\title{
A derivative-free multistart framework for an automated noncoplanar beam angle
} optimization in IMRT

\author{
Humberto Rocha and Joana Dias \\ FEUC and Inesc-Coimbra, University of Coimbra, Portugal \\ Tiago Ventura \\ Medical Physics Department, IPOCFG,EPE, Coimbra, \\ Portugal and Physics Department, University of Aveiro, Portugal \\ Brígida Ferreira \\ School of Allied Health Technologies, Polytechnic of Porto, \\ Porto, Portugal and I3N, University of Aveiro, Portugal \\ Maria do Carmo Lopes \\ Medical Physics Department, IPOCFG,EPE, Coimbra, Portugal
}

( Dated: July 26, 2016)

Purpose: The inverse planning of an intensity-modulated radiation therapy (IMRT) treatment requires decisions regarding the angles used for radiation incidence, even when arcs are used. The possibility of improving the quality of treatment plans by an optimized selection of the beam angle incidences - beam angle optimization (BAO) - is seldom done in clinical practice. The inclusion of noncoplanar beam incidences in an automated optimization routine is even more unusual. However, for some tumor sites, the advantage of considering noncoplanar beam incidences is well known. This paper presents the benefits of using a derivative-free multistart framework for the optimization of the noncoplanar BAO problem.

Methods: Multistart methods combine a global strategy for sampling the search space with a local strategy for improving the sampled solutions. The proposed global strategy allows a thorough exploration of the continuous search space of the highly non-convex BAO problem. To avoid local entrapment, a derivative-free method is used as local procedure. Additional advantages of the derivative-free method include the reduced number of function evaluations required to converge and the ability to use multi-threaded computing. Twenty nasopharyngeal clinical cases were selected to test the proposed multistart framework. The planning target volumes included the primary tumor, the high and low risk lymph nodes. Organs-at-risk included the spinal cord, brainstem, optical nerves, chiasm, parotids, oral cavity, brain, thyroid, among others. For each case, a setup with seven equispaced beams was chosen and the resulting treatment plan, using a multicriteria optimization framework, was then compared against the coplanar and noncoplanar plans using the optimal beam setups obtained by the derivative-free multistart framework.

Results: The optimal noncoplanar beam setup obtained by the derivative-free multistart framework lead to high quality treatment plans with better target coverage and with improved organ sparing compared to treatment plans using equispaced or optimal coplanar beam angle setups. The noncoplanar treatment plans achieved, e.g., an average reduction in the mean dose of the oral cavity of $6.1 \mathrm{~Gy}$ and an average reduction in the maximum dose of the brainstem of 7 Gy when compared to the equispaced treatment plans.

Conclusions: The noncoplanar BAO problem is an extremely challenging multi-modal optimization problem that can be successfully addressed through a thoughtful exploration of the continuous highly non-convex BAO search space. The proposed framework is capable of calculating high quality treatment plans and thus can be an interesting alternative towards automated noncoplanar beam selection in IMRT treatment planning which is nowadays the natural trend in treatment planning.

Keywords: IMRT Treatment Planning, Noncoplanar Beam Angle Optimization, Derivative-free Optimization, Multistart 


\section{INTRODUCTION}

Many of the recent studies on the optimal selection of beam angle incidences - beam angle optimization (BAO) - for intensity-modulated radiation therapy (IMRT) have considered noncoplanar beam directions ${ }^{1-12}$. These studies have demonstrated a substantial plan's quality improvement by the inclusion of noncoplanar beam incidences, specially for intra-cranial tumor sites ${ }^{1}$. Recently, the use of noncoplanar beam angles in volumetric modulated arc therapy (VMAT) was also proposed to combine the benefits of arc therapy, such as short treatment times, with the benefits of noncoplanar IMRT plans, such as improved organ sparing ${ }^{11,12}$. Selected noncoplanar beam angle directions can be used as anchor points of the arc therapy trajectory ${ }^{11}$ which is yet another reason for the improvement of automated selection of optimal noncoplanar beam angle directions that can prove to be important in extra-cranial tumor sites as well ${ }^{13,14}$. In clinical practice, in contrast, equispaced coplanar irradiation beam directions are still commonly used. Alternatively, directions are manually selected by the treatment planner on a long trial-and-error process since commercial treatment planning systems have none or very few resources available for optimal selection of beam angle incidences. One of the reasons for this limited commercial offer is the difficulty of solving the BAO problem, a highly non-convex multi-modal optimization problem on a large search space ${ }^{15}$.

The BAO approaches can be separated into two different classes. The first class addresses sequentially the selection of beam irradiation directions, BAO problem, and the fluence map optimization (FMO) problem. Dosimetric surrogates or geometric features are considered as measures of the beam ensembles quality for driving the BAO problem ${ }^{2,9,16}$. The second class addresses simultaneously the BAO and FMO problems. The measure of the beam ensembles quality used to drive the BAO problem is the optimal solution of the FMO problem ${ }^{5,10,11,15,17-21}$. In this second class, for most of the $\mathrm{BAO}$ approaches, a discrete sample of all possible beam irradiation directions is considered and the $\mathrm{BAO}$ problem is modeled as a combinatorial optimization problem. The best ensemble of $n$-beam irradiation directions among the discrete set of possible directions is obtained by performing exhaustive searches guided by a variety of different methods including gradient search ${ }^{15}$, neighborhood search ${ }^{17}$, branch-and-prune ${ }^{18}$, hybrid , genetic algorithms ${ }^{20}$ or simulated annealing ${ }^{21}$. This combinatorial formulation of the BAO leads to an NP (Nondeterministic Polynomial time) hard problem, i.e. there is no algorithm known able to find, in a polynomial run time, the optimal solution of the combinatorial $\mathrm{BAO}$ problem ${ }^{22}$. Alternatively, iterative $\mathrm{BAO}^{3,5-7,11,12}$ sequentially adds beams, one at a time, to a treatment plan, significantly reducing the number of beam combinations while achieving similar treatment plan quality ${ }^{1}$. For an ensemble with $n-1$ irradiation beam directions, the $n$th

beam direction selected by testing individually each of the possible remaining directions combined with the $n-1$ $n-1$ beam direction ensemble. Nevertheless, if a large discrete pool of beam directions is considered, iterative BAO remains computationally expensive. For nasopharyngeal tumor cases, e.g., about 1400 beam orientations are available for a $5^{\circ}$ angular spacing, excluding caudal beams which could result in a collision of the couch and/or patient with the gantry ${ }^{12}$. Finding the best ensemble of 7 beams in 1400 candidate beams through iterative BAO still requires 9779 FMOs.

In this paper, we propose a completely different methodological approach for the noncoplanar BAO in IMRT by exploring the continuous search space of the highly non-convex BAO problem through a parallel multistart derivative-free framework. Multistart methods with local search procedures are globally convergent and its interest and application fields continue to rise ${ }^{23}$. The proposed multistart framework combines a global strategy for a thoughtful sampling of the search space with a local strategy for improving the sampled solutions that avoids local entrapment by using a derivative-free algorithm. A set of twenty clinical cases of nasopharyngeal tumors treated at the Portuguese Institute of Oncology of Coimbra (IPOC) is used to discuss the benefits of the novel approach proposed for the BAO problem.

\section{MATERIAL AND METHODS}

\section{A. Mathematical formulation of the noncoplanar BAO problem}

The mathematical formulation of the noncoplanar BAO problem considers all continuous beam irradiation directions. Let $n$ denote the fixed number of noncoplanar beam directions, $\theta$ denote a gantry angle and $\phi$ denote a couch angle. Note that, the coplanar beam angles commonly used in clinical practice are obtained for a fixed couch position at $\phi=0$. Instead of a discretized sample, continuous gantry angles, $\theta \in[0,360]$, and couch angles, $\phi \in[-90,90]$, are considered. A simple formulation for the BAO problem is obtained by selecting an objective function such that the 


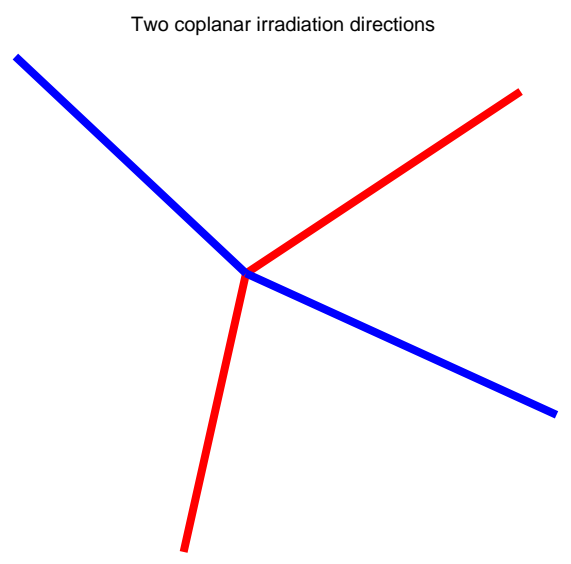

(a)

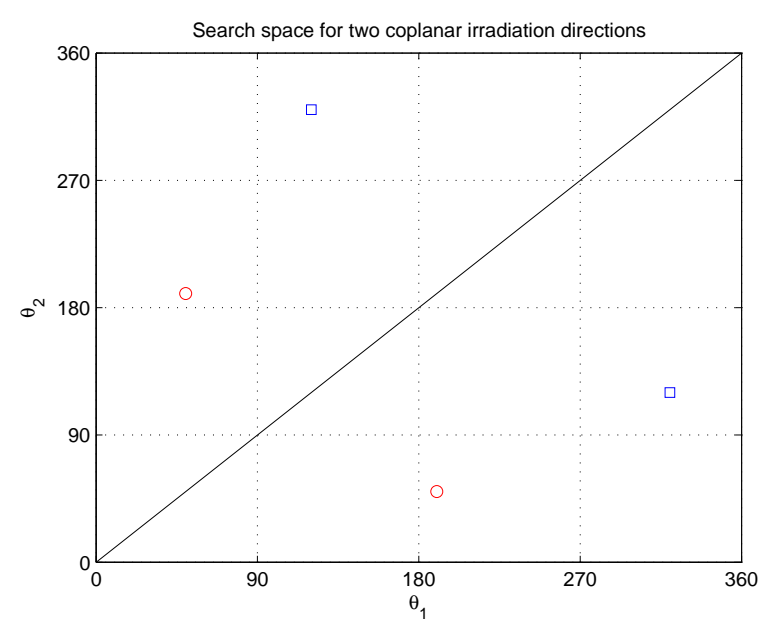

(b)

FIG. 1. 2-beam ensembles of coplanar incidences - 1(a) and corresponding solutions in the search space $[0,360]^{2}-1(\mathrm{~b})$.

best set of beam angles is obtained for the function's minimum:

$$
\begin{aligned}
& \min f\left(\left(\theta_{1}, \phi_{1}\right), \ldots,\left(\theta_{n}, \phi_{n}\right)\right) \\
& \text { s.t. }\left(\left(\theta_{1}, \phi_{1}\right), \ldots,\left(\theta_{n}, \phi_{n}\right)\right) \in[0,360]^{n} \times[-90,90]^{n} .
\end{aligned}
$$

The objective function $f\left(\left(\theta_{1}, \phi_{1}\right), \ldots,\left(\theta_{n}, \phi_{n}\right)\right)$ that measures the quality of the set of beam directions $\left(\theta_{1}, \phi_{1}\right), \ldots$, $\left(\theta_{n}, \phi_{n}\right)$ corresponds to the optimal value of the FMO problem for each fixed set of beam directions and incorporates information concerning the angles for which collisions between the patient/couch and the gantry may occur:

$$
f\left(\left(\theta_{1}, \phi_{1}\right), \ldots,\left(\theta_{n}, \phi_{n}\right)\right)= \begin{cases}+\infty & \text { if collisions occur } \\ \text { optimal value of the FMO } & \text { otherwise }\end{cases}
$$

\section{B. Multistart parallel framework for noncoplanar BAO}

\section{Choice of the starting solutions}

An important feature of a $\mathrm{BAO}$ problem solution (beam ensemble) is the simple fact that the order of the beam irradiation directions is irrelevant. E.g. for 2-beam coplanar ensembles, ordered pairs $(50,190)$ and $(120,320)$ correspond to the same solutions of the BAO problem as ordered pairs $(190,50)$ and $(320,120)$, respectively. This symmetry of the search space is illustrated in Fig. 1 where coplanar beam directions displayed in Fig. 1(a) have 2 symmetric solutions in the search space $[0,360]^{2}$ displayed in Fig. 1(b).

Typically, multistart methods sample the search space by considering starting points selected randomly ${ }^{23}$. For a BAO multistart strategy, the symmetry of the search space illustrated by the diagonal line in Fig. 1(b) implies that points in opposite sides of the diagonal line might be searching for the exact same solutions. Thus, the symmetry feature of the continuous search space must be taken into account when selecting starting points and throughout the optimization procedure. The simple strategy of sorting all the solutions during the optimization process solves this issue and leads to a huge reduction of the search space. E.g. for the 2-beam coplanar ensemble example of Fig. 1(b), we guarantee that we are only searching in one side of the diagonal line and, with that, we reduce the search space to half. In general, for $n$-beam noncoplanar ensembles, this strategy reduces the search space by $2^{n}$. Thus, for the 5-, 7- or 9-beam noncoplanar directions optimization problems, the search space is only $3.13 \%$ of $[0,360]^{5} \times[-90,90]^{5}$, $0.78 \%$ of $[0,360]^{7} \times[-90,90]^{7}$ and $0.19 \%$ of $[0,360]^{9} \times[-90,90]^{9}$, respectively.

For a BAO multistart strategy, the starting solutions should sample the search space $[0,360]^{n} \times[-90,90]^{n}$ thoroughly. The rationale behind the choice of the sorted starting solutions must acknowledge the fact that the search space is 
the and has a peculiar shape. Thus, we must assure that the starting solutions belong to the reduced search space and simultaneously are well spread attempting to cover the search space as best as possible.

The strategy developed consists in dividing the gantry beam directions, $\theta \in[0,360]$, in 4 quadrants $\left(Q_{1}-Q_{4}\right)$ and the couch beam directions, $\phi \in[-90,90]$, in 2 quadrants $\left(Q_{4}, Q_{1}\right)$. For a full comprehension of the strategy sketched, the 2- and 3-beam coplanar cases are first introduced, cases where graphical illustration is straightforward. There are ten and twenty possible (sorted) distributions by the four quadrants of the 2-beam and 3-beam coplanar ensembles, respectively. Examples of these distributions are displayed in Fig. 2. Fig. 2(a) and Fig. 2(c) display examples of 2-beam and 3-beam ensembles for each one of the ten and twenty cases, respectively. Fig. 2(b) and Fig. 2(d) display the corresponding painted regions (squares/cubes) of the reduced search space. Note that, despite the squares crossed by the diagonal line are all painted in Fig. 2(b), sorted points are only in one side of the line. A possible sampling of the starting solutions, assuring that they belong to the reduced search space while covering well the reduced search space, can be obtained by considering one starting solution for each of these regions. For $n=2$, 10 starting points are considered, corresponding to 10 squares in the reduced search space (out of 16 for the entire search space). For $n=3$, selecting one starting point for each one of the painted cubes in the reduced search space, 20 starting points are considered (out of 64 for the entire search space). Note that, for $n=2$ the reduced search space is $50 \%$ of the entire search space while for $n=3$ the reduced search space is $25 \%$ of the entire search space corresponding to a larger reduction for higher dimensions. However, the number of regions of the reduced search space for $n=2$ is only 10 compared to the 20 cubes for $n=3$. Thus, the number of regions of the reduced search space increases as the number of beams increases. Furthermore, the dimension of the regions increases as well. In general, for $n$-beam coplanar directions, the total number of hypercubes of the entire search space is $4^{n}$ while the number of

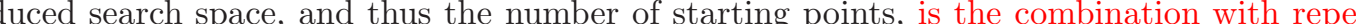
of $\left(\begin{array}{c}n+4-1 \\ 4\end{array}\right)=\frac{(n+4-1) !}{(4-1) ! n !}$. Therefore, for the 5-, 7- or 9-beam coplanar ensembles optimization problems, the reduced search space has 56, 120 and 220 hypercubes, compared to 1024, 16384 and 262144 hypercubes for the entire search space, respectively.

The extension of this strategy to the noncoplanar case requires the inclusion of the couch beam directions, $\phi \in$ are 4 different possibilities of distribution of the couch angles by the 2 quadrants while for $n=3$ there are 8 different possibilities of distribution of the couch angles for each possible sorted coplanar ensemble. In general, for $n$-beam noncoplanar ensembles, for each possible sorted coplanar ensemble, there are $2^{n}$ different possibilities of distribution of the couch angles by the 2 quadrants. Thus, for $n$-beam noncoplanar ensembles, the total number of hypercubes of the entire search space is $4^{n} \times 2^{n}$ while the number of hypercubes of the reduced search space is $\left(\begin{array}{c}n+4-1 \\ 4\end{array}\right) \times 2^{n}$ $=\frac{(n+4-1) ! \times 2^{n}}{(4-1) ! n !}$. Therefore, for the 5-, 7- or 9-beam noncoplanar ensembles optimization problems, the reduced search space has 1792, 15360 and 112640 hypercubes, compared to 32768, 2097152 and 134217728 hypercubes for the entire search space, respectively.

For the noncoplanar case, considering one starting solution for each of the hypercubes of the reduced space, in a coplanar case, would assure that initial points belong to the reduced search space while covering well the reduced search space. However, such strategy considers a large number of initial points and consequently many FMO calculations which increases the total computational time. Notice that coplanar solutions lay on the frontier of different hypercubes. Following the directions of the positive basis used in the local search procedure, neighbor hypercubes can be reached when considering coplanar beam angle ensembles as initial solutions. Thus, coplanar beam angle ensembles are selected as initial solutions so that the local search procedure assures that all hypercubes of the reduced search space can be explored.

\section{Local search procedure}

We have shown, in previous works, that a beam angle ensemble can be improved in a continuous manner using derivative-free algorithms ${ }^{24-26}$. Thus, pattern search methods (PSM) are used in this framework, as local search procedure, since they avoid local entrapment and require few FMO calculations to converge ${ }^{24-26}$. PSM are derivativefree directional search methods that generate, iteratively, a sequence of non-increasing iterates following the directions of positive bases to move towards solutions that can decrease the objective function value. A positive basis for the search space is a set of directions (vectors) whose positive combinations span the entire search space, but no proper set does. It can be proved that, for an $n$-dimensional space, a positive basis contains at most $2 n$ directions and

ot contain less than $n+1$ directions 27,28 . Positive bases with $2 n$ and $n+1$ elements are referred to as maximal and minimal positive basis, respectively. Maximal and minimal positive bases commonly used are $[I-I]$, with $I$ being the identity matrix of dimension $n$, and $[I-e]$ with $e=[1 \ldots 1]^{\top}$, respectively.

Typically, PSM are organized around two steps at every iteration ${ }^{24-26}$. In the first step, called search step, a finite 


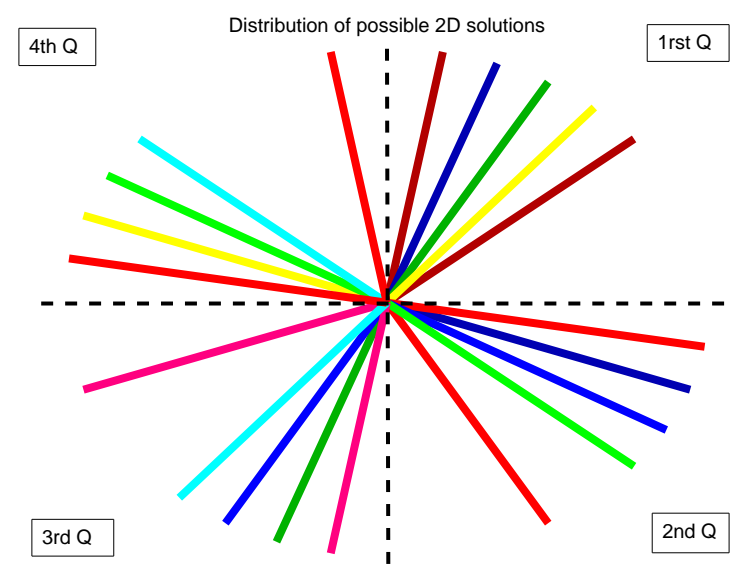

(a)

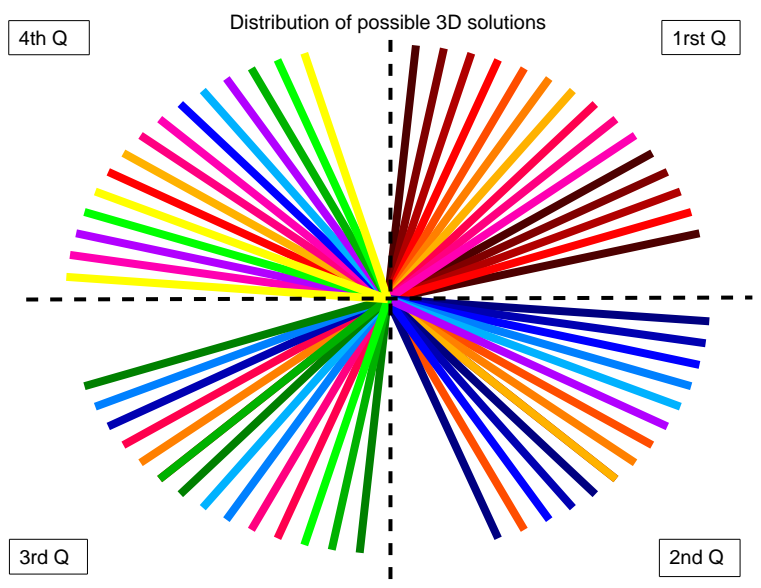

(c)

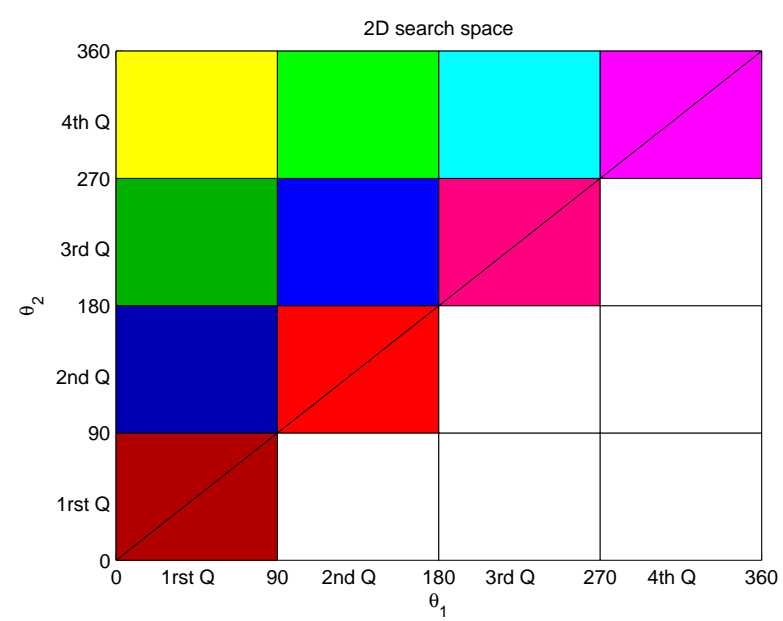

(b)

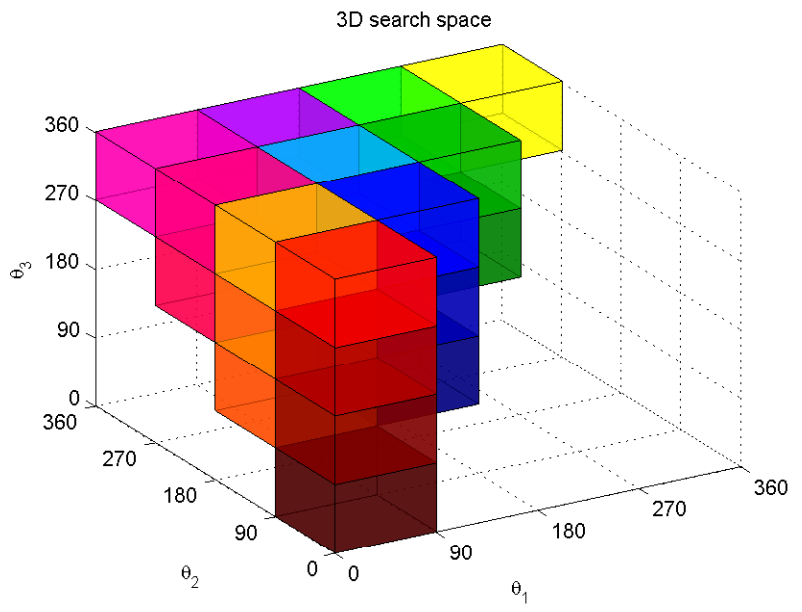

(d)

FIG. 2. Possible 2-beam coplanar ensembles distribution by the four quadrants - 2(a) and corresponding squares in the search space $[0,360]^{2}-2(\mathrm{~b})$. Possible 3-beam coplanar ensembles distribution by the four quadrants $-2(\mathrm{c})$ and the corresponding cubes in the search space $[0,360]^{3}-2(d)$.

search is performed using any strategy, heuristic or algorithm attempting to find a solution that improves the objective function value of the current best solution. This step, free of rules, allows searches away from the neighborhood of the current best solution, providing flexibility for a global search. If the search step fails to improve the current objective function value, a second step, called poll step, is performed in the neighborhood of the current best solution. The properties of positive bases are used in the poll step to perform a local search around the the current best solution. A key motivation for the use of positive bases is that, unless the current best solution is at a stationary point, at least one vector (direction) of a positive basis is a descent direction, i.e., following that direction leads to an objective function reduction for a sufficiently small step-size. Therefore, if the poll step fails to accomplish a reduction of the current objective function value, and thus the iteration is unsuccessful, the step-size is decreased. On the other hand, if the the value of the objective function is decreased during the poll step, the step-size is increased or held constant. This iterative procedure ends when the step-size becomes too small or when the maximum number of function evaluations is reached. Thus, the step-size has two purposes beyond its use as stopping criteria: to bound the size of the minimization step and also to control the local area where the function is sampled around the current best solution.

PSM have the ability to converge globally, i.e., from arbitrary points, to local minimizers ${ }^{29}$. Furthermore, PSM have the ability to avoid local entrapment. Gathering these two features make PSM a good choice for the local search procedure to be embedded in our parallel multistart framework. 
Multistart methods are organized around two phases that are typically designated as global and local phases ${ }^{23}$. In the first phase, the global phase, the search space is sampled by a given number of selected starting points where the objective function is evaluated. Then, in the second phase, the starting points outcome is locally improved by local search procedures. A major drawback of multistart methods is the multiple discovery of the same local minima, i.e., the outcome of local procedures originated in different starting points may correspond to the same local minima leading to precious computational time waste. For a parallel setting, this risk increases when the same region of the search space is simultaneously searched by local procedures originated from different regions. There are many different strategies to avoid local search overlap and repetitive discovery of the same minima including clustering methods ${ }^{30}$ or regions of attraction of a local minimum ${ }^{31}$. The notion of regions of attraction can be used to avoid multiple discovery of the same minima and simultaneously to accelerate the search procedure. For the BAO problem, given a local search procedure $\mathcal{L} \mathcal{S}$, the region of attraction of a local minimum $\mathbf{x}^{*}$ can be defined as:

$$
A=\left\{\mathbf{x} \in[0,360]^{n} \times[-90,90]^{n}: \mathcal{L S}(\mathbf{x})=\mathbf{x}^{*}\right\}
$$

Thus, $A$ is the region of points $\mathbf{x} \in[0,360]^{n} \times[-90,90]^{n}$ whose local search procedure outcome, $\mathcal{L} \mathcal{S}(\mathbf{x})$, is the same local minimum $\mathbf{x}^{*}$. The strategy of allowing only one local search for each region of attraction can prevent overlap of local searches and with that repetitive discovery of the same minima. However, regions of attraction can hardly be determined in practice. Instead, regions of attraction are usually stochastically defined as the set of points in a neighborhood of the local minimum $\mathbf{x}^{*}$, i.e. the set of points whose distance is inferior to a certain radius $R_{A}{ }^{31}$ :

$$
A=\left\{\mathbf{x} \in[0,360]^{n} \times[-90,90]^{n}:\left\|\mathbf{x}-\mathbf{x}^{*}\right\|<R_{A}\right\}
$$

For a sequential multistart procedure, the computation of a first local minimum allows the definition of its region of attraction and, thereafter, it is possible to decide stochastically if a subsequent iterate belongs to the region of attraction. However, for a parallel setting, a different rationale is required since many or all local search procedures coexist in time. For the noncoplanar BAO parallel multistart approach the stochastic region of attraction (3) is generalized by considering the $l_{\infty}$-norm instead of the Euclidean norm, $l_{2}$-norm, centered at the midpoints $M$ of all hypercubes of the reduced noncoplanar search space instead of at local minima $\mathbf{x}^{*}$ :

$$
A_{B A O}=\left\{\mathbf{x} \in[0,360]^{n} \times[-90,90]^{n}:\|\mathbf{x}-M\|_{\infty}<R_{A}\right\} .
$$

For the noncoplanar BAO, instead of circles or balls, with this definition of regions of attraction we have hypercubes that, choosing $R_{A}=45$, for the coplanar cases $n=2$ and $n=3$ correspond exactly to the squares and cubes painted in Figs. 2(b) and 2(d), respectively.

A tailored strategy was sketched to address the noncoplanar $n$-beam BAO problem. In the first iteration, $N_{c}=\frac{(n+4-1) !}{(4-1) ! n !}$ initial coplanar beam ensembles, $\mathbf{x}_{i}^{0} \in[0,360]^{n} \times 0^{n}, i=1, \ldots, N_{c}$, are chosen considering all sorted combinations of the gantry's 4 quadrants, corresponding, for $n=2$ and $n=3$, to points in the painted squares and cubes in Figs. 2(b) and 2(d), respectively. Then, the objective function value is evaluated for each one of the initial beam ensembles and the best beam ensemble $\mathbf{x}^{*}$ is determined, corresponding to the best objective function value $f^{*}=f\left(\mathbf{x}^{*}\right)=\min \left\{f\left(\mathbf{x}_{1}^{0}\right), f\left(\mathbf{x}_{2}^{0}\right), \ldots, f\left(\mathbf{x}_{N_{c}}^{0}\right)\right\}$. The best objective function values and corresponding coplanar solutions calculated for some of the regions (hypercubes) of the noncoplanar reduced search space are stored: $\mathbf{x}_{i}^{\text {best }}=\mathbf{x}_{i}^{0}, i=1, \ldots, N_{c} ; f_{i}^{b e s t}=f\left(\mathbf{x}_{i}^{0}\right), i=1, \ldots, N_{c}$. Note that the total number of hypercubes, $N_{n c}=\frac{(n+4-1) ! \times 2^{n}}{(4-1) ! n !}$, of the noncoplanar reduced search space is larger than the initial coplanar beam ensembles. Thus, the objective function value for the hypercubes where no solution was yet computed is set to $+\infty, f_{i}^{b e s t}=+\infty, i=N_{c}+1, \ldots, N_{n c}$. Moreover, only a subset of all hypercubes of the noncoplanar reduced search space will have "active" local search procedures. In order to store the information of which regions have active local search procedures at each iteration, a boolean vector Active A $_{n c} \times 1$ is defined.

A parallel local search procedure using PSM is performed in the following iterations for each of the regions with active local search procedures. For the noncoplanar BAO problem, we choose the initial step-size as a power of 2 , 32, and we use a common update of the step-size that is halved at unsuccessful iterations and held constant at successful ones. For this selection of the initial search-step, since the initial solutions are vectors of integers, all solutions will be integers until the step-size becomes inferior to 1 which will be the stopping criteria. For the noncoplanar BAO problem, we choose the maximal positive basis, $\left[\begin{array}{ll}I-I\end{array}\right]$. The directions of the maximal positive basis correspond 
205 to the rotation of each individual incidence direction (gantry or couch angle) clockwise and counter-clockwise, for positive and negative directions, respectively. Thus, with the selected initial coplanar beam ensembles and the set of directions of the positive basis considered, PSM can reach all the different hypercubes of the noncoplanar reduced search space and perform local searches there. At iteration $k$, the local search outcome in active region $i$ is one of the three possible outcomes:

- The local search is successful, i.e. $f\left(\mathbf{x}_{i}^{k}\right)<f_{i}^{b e s t}$, and $\mathbf{x}_{i}^{k}$ remains in region $i$. In this case, the best objective function value and best solution in region $i$ are updated to the new function value and the new iterate.

- The local search is successful but $\mathbf{x}_{i}^{k}$ is outside of region $i$. In this case, region $i$ becomes inactive, Active $\mathbf{e}_{i}=0$, since local search pointed outside the region. The successful solution $\mathbf{x}_{i}^{k}$ belongs to a region $j \neq i$ where local search may be active or not leading to four different possibilities:

1. If region $j$ has no active local search, Active $_{j}=0$ :

(a) If the objective function at $\mathbf{x}_{i}^{k}$ improves the best objective function value of region $j$ then region $j$ becomes active and iterate and function values of region $j$ are updated.

(b) If the objective function at $\mathbf{x}_{i}^{k}$ does not improve the best objective function value of region $j$, that local search ends.

2. If a local search is active in region $j, \mathbf{A c t i v e}_{j}=1$ :

(a) If the objective function at $\mathbf{x}_{i}^{k}$ improves the best objective function value of region $j$ then region $j$ remains active and iterate, function and step-size values of region $j$ are updated.

(b) If the objective function at $\mathbf{x}_{i}^{k}$ does not improve the best objective function value of region $j$ then region $j$ remains active continuing the local search process existent there.

Thus, when a local search is directed to a different region, the number of active local searches is decremented by one in three out of the four possibilities.

- The local search is not successful. In this case, the step-size is decreased. When the step-size becomes inferior to the minimum step-size then the local search ends.

This process is repeated while regions with active local search procedures still exists. For computational time reasons, only the most promising regions are further explored. Therefore, local search procedure in region $i$ remains active, Active $_{i}=1$, if the objective function at $\mathbf{x}_{i}^{\text {best }}$ is not worst than the best objective function value $f^{*}$ within a defined threshold $p \geq 0$, i.e., if $f\left(x_{i}^{\text {best }}\right) \leq(1+p) f^{*}$. Otherwise, Active $_{i}=0$. The different local search procedures can always progress towards regions whose local procedures are not currently active. We are able to describe now the parallel multistart algorithm:

\section{Parallel multistart algorithm framework}

\section{Initialization:}

- Choose $\mathbf{x}_{i}^{0} \in[0,360]^{n} \times 0^{n}, i=1, \ldots, N_{c}$;

- Evaluate in parallel the objective function value at these $N_{c}$ points;

- Determine the best objective function value $f^{*}=f\left(\mathbf{x}^{*}\right)=\min \left\{f\left(\mathbf{x}_{1}^{0}\right), f\left(\mathbf{x}_{2}^{0}\right), \ldots, f\left(\mathbf{x}_{N_{c}}^{0}\right)\right\}$ and the corresponding best initial beam ensemble $\mathbf{x}^{*}$;

- Set $\mathbf{x}_{i}^{\text {best }} \leftarrow \mathbf{x}_{i}^{0}, i=1, \ldots, N_{c}, f_{i}^{b e s t} \leftarrow f\left(\mathbf{x}_{i}^{0}\right), i=1, \ldots, N_{c}$ and $f_{i}^{b e s t} \leftarrow+\infty, i=N_{c}+1, \ldots, N_{n c}$;

- Set Active $_{i} \leftarrow 1, i=1, \ldots, N_{c}$, Active $_{i} \leftarrow 0, i=N_{c}+1, \ldots, N_{n c}$;

- Set $k \leftarrow 1$;

- Choose $p \geq 0$, a positive spanning set, $\sigma_{i}^{1}>0, i=1, \ldots, N$ and $\sigma_{\min }$;

\section{Iteration:}

1. Perform local search in parallel for the active regions using PSM; 


\section{For each active region $i$ do}

If local search is successful, i.e. $f\left(\mathbf{x}_{i}^{k}\right)<f\left(\mathbf{x}_{i}^{k-1}\right)$ then

If $\mathbf{x}_{i}^{k}$ remains in region $i$ then defined for the primary tumor (PTV-T) and a lower dose level (59.4Gy) was defined for the lymph nodes (PTV-N). Several auxiliary structures were constructed by computerized volume expansions to support the dose optimization. To prevent possible high doses in the lymph nodes, PTV-N shell was created by subtracting a $10 \mathrm{~mm}$ margin of

Treatment plan optimization is inherently a multicriteria procedure. Nevertheless, typically, the FMO problem is modeled as a weighted sum function with conflicting objectives. Moreover, constraints are many times implemented as objectives, which difficult the trade-off between objectives without violating constraints. The multicriteria approaches that have been proposed for the FMO problem can be divided into two classes. In the first class, treatment plans are selected a posteriori from a set of Pareto-optimal treatment plans ${ }^{32,33}$. In the second class, a set of criteria (constraints and objectives) that have to be met during the multicriteria optimization procedure is defined a priori ${ }^{5,34,35}$. For a fully automated noncoplanar BAO procedure, this second class of multicriteria approaches is a straightforward option. Thus, we choose a multicriterial optimization based on a set of criteria (radiation dose prescription) called wish-list ${ }^{5,34,35}$ to address the FMO problem.

The wish-list constructed for the clinical examples of nasopharyngeal tumors treated at IPOC is given in Table I. The nasopharyngeal tumors are complex cases due to the large number of sensitive organs in this region which increases the difficulty of the radiotherapy treatment planning. The structures in the wish-list include the planning target volume (PTV), tumor to be treated plus some safety margins, and several organs at risk (OARs): brainstem, spinal cord, lens, retinas, optics (optical nerves + chiasm), ears, pituitary gland, oral cavity, parotids, mandible, temporomandibular joints (TMJ), larynx, esophagus, thyroid, brain and lungs. The prescribed and tolerance doses were defined according to the protocols defined for nasopharyngeal tumors at IPOC. A higher dose level (70Gy) was PTV-T to PTV-N. Ring PTV-T and Ring PTV-N were created with $10 \mathrm{~mm}$ of thickness at $10 \mathrm{~mm}$ distance from PTV-N and PTV-T, respectively, to improve target coverage and conformity, respectively. External Ring, a ring of $10 \mathrm{~mm}$ thickness, was created next to the patient outer contour to prevent possible high values of dose entrance.

The wish-list contains 11 hard constraints and 28 prioritized objectives based on the prescribed and tolerance doses for all the structures considered in the optimization. All hard constraints are maximum-dose constraints. Hard 
TABLE I. Wish-list for nasopharyngeal tumor cases.

\begin{tabular}{|c|c|c|c|c|c|c|}
\hline & Structure & Type & \multicolumn{4}{|c|}{ Limit } \\
\hline \multirow{12}{*}{ Constraints } & PTV-N & maximum & \multicolumn{4}{|c|}{$63.6 \mathrm{~Gy}(=107 \%$ of prescribed dose) } \\
\hline & PTV-T & maximum & \multicolumn{4}{|c|}{74.9 Gy ( $=107 \%$ of prescribed dose) } \\
\hline & PTV-N shell & maximum & \multicolumn{4}{|c|}{$63.6 \mathrm{~Gy}(=107 \%$ of prescribed dose) } \\
\hline & Spinal cord & maximum & \multicolumn{4}{|c|}{45 Gy } \\
\hline & Brainstem & maximum & \multicolumn{4}{|c|}{$54 \mathrm{~Gy}$} \\
\hline & Optics & maximum & \multicolumn{4}{|c|}{$55 \mathrm{~Gy}$} \\
\hline & Retinas & maximum & \multicolumn{4}{|c|}{$45 \mathrm{~Gy}$} \\
\hline & Ring PTV-N & maximum & \multicolumn{4}{|c|}{50.5 Gy $(=85 \%$ of prescribed dose) } \\
\hline & Ring PTV-T & maximum & \multicolumn{4}{|c|}{59.5 Gy ( $=85 \%$ of prescribed dose) } \\
\hline & External Ring & maximum & \multicolumn{4}{|c|}{$45 \mathrm{~Gy}$} \\
\hline & Body & maximum & \multicolumn{4}{|c|}{$70 \mathrm{~Gy}$} \\
\hline & Structure & Type & Priority & Goal & Sufficient & Parameters \\
\hline \multirow{28}{*}{ Objectives } & PTV-N & LTCP & 1 & 1 & 0.5 & $P_{D}=59.4 \mathrm{~Gy} ; \tau=0.75$ \\
\hline & PTV-T & LTCP & 2 & 1 & 0.5 & $P_{D}=70 \mathrm{~Gy} ; \quad \tau=0.75$ \\
\hline & PTV-N shell & LTCP & 3 & 1 & 0.5 & $P_{D}=59.4 \mathrm{~Gy} ; \tau=0.75$ \\
\hline & External ring & maximum & 4 & $42.75 \mathrm{~Gy}$ & - & - \\
\hline & Spinal cord & maximum & 5 & $42.75 \mathrm{~Gy}$ & - & - \\
\hline & Brainstem & maximum & 6 & $51.3 \mathrm{~Gy}$ & - & - \\
\hline & Optics & maximum & 7 & $52.25 \mathrm{~Gy}$ & - & - \\
\hline & Retinas & maximum & 8 & $42.75 \mathrm{~Gy}$ & - & - \\
\hline & Lens & gEUD & 9 & $12 \mathrm{~Gy}$ & - & $a=12$ \\
\hline & Ears & mean & 10 & $50 \mathrm{~Gy}$ & - & - \\
\hline & Parotids & mean & 11 & $50 \mathrm{~Gy}$ & - & - \\
\hline & Oral cavity & mean & 12 & $45 \mathrm{~Gy}$ & - & - \\
\hline & TMJ & maximum & 13 & $66 \mathrm{~Gy}$ & - & - \\
\hline & Mandible & maximum & 14 & $66 \mathrm{~Gy}$ & - & - \\
\hline & Esophagus & mean & 15 & $45 \mathrm{~Gy}$ & - & - \\
\hline & Larynx & mean & 16 & $45 \mathrm{~Gy}$ & - & - \\
\hline & Optics & gEUD & 17 & $48 \mathrm{~Gy}$ & - & $a=12$ \\
\hline & Retinas & gEUD & 18 & $22 \mathrm{~Gy}$ & - & $a=12$ \\
\hline & Lens & gEUD & 19 & 6 Gy & - & $a=12$ \\
\hline & Ears & mean & 20 & $45 \mathrm{~Gy}$ & - & - \\
\hline & Parotids & mean & 21 & $26 \mathrm{~Gy}$ & - & - \\
\hline & Oral cavity & mean & 22 & $35 \mathrm{~Gy}$ & - & - \\
\hline & Esophagus & mean & 23 & $35 \mathrm{~Gy}$ & - & - \\
\hline & Larynx & mean & 24 & $35 \mathrm{~Gy}$ & - & - \\
\hline & Brain & gEUD & 25 & 54 Gy & - & $a=12$ \\
\hline & Pituitary gland & gEUD & 26 & $60 \mathrm{~Gy}$ & - & $a=12$ \\
\hline & Thyroid & mean & 27 & $27.5 \mathrm{~Gy}$ & - & - \\
\hline & Lungs & mean & 28 & 5 Gy & - & - \\
\hline
\end{tabular}

constraints must be strictly met while objectives are optimized following the priorities defined in the wish-list. The higher an objective priority, the most likely the corresponding objective will be fulfilled. The maximum dose was considered for serial organs, e.g. for the brainstem or the spinal cord. For parallel organs, e.g. parotids or thyroid, the mean dose was considered. For some other organs, a generalized Equivalent Uniform Dose $(g E U D)$ objective was considered $^{5}$,

$$
g E U D=T_{f}\left(\frac{1}{V} \sum_{k} D_{k}^{a}\right)^{\frac{1}{a}}
$$

where $T_{f}$ is the number of treatment fractions, $V$ the number of voxels of the discretized structure, $D_{k}$ the dose in voxel $k$ and $a$ is the tissue-specific parameter that describes the volume effect. We considered $a=12$ attempting to minimize the volume of a given OAR with a high dose. Note that for $a=1,0,+\infty$ and $-\infty, g E U D$ is equivalent to the arithmetic mean, geometric mean, maximum and minimum doses, respectively.

The logarithmic tumor control probability $(L T C P)$ was considered for the target dose optimization ${ }^{5}$,

$$
L T C P=\frac{1}{V_{T}} \sum_{k=1}^{V_{T}} e^{-\tau\left(D_{k}-P_{D}\right)},
$$

where $V_{T}$ is the number of voxels in the target structure, $D_{k}$ is the dose in voxel $k, P_{D}$ is the prescribed dose, and $\tau$ is the cell sensitivity parameter. For doses $D_{k}$ lower than the prescribed dose $P_{D}, L T C P$ has an exponential penalty. For doses higher than the prescribed dose, $L T C P$ slowly approaches 0 . Note that our goal is an $L T C P$ equal to one which would correspond to an homogeneous dose equal to $P_{D}$. The tumor coverage can be improved by increasing the value of $\tau$ which corresponds to a decrease in the number of voxels with a low dose.

A primal-dual interior-point algorithm tailored for multicriteria IMRT treatment planning, named $2 p \epsilon c^{34}$, was used for the optimization of the FMO problem using the described wish-list. The $2 p \epsilon c$ multicriteria algorithm generate, in 
TABLE II. Gantry and couch angles obtained for $B A O_{n c}$ and $B A O_{c}$ treatment plans.

\begin{tabular}{|c|c|c|c|c|c|c|c|}
\hline Patient & Angle & $B A O_{n c}$ & $B A O_{c}$ & Patient & Angle & $B A O_{n c}$ & $B A O_{c}$ \\
\hline 1 & $\begin{array}{l}\text { Gantry } \\
\text { Couch }\end{array}$ & $\begin{array}{c}(38,97,115,194,240,301,349) \\
(344,358,292,16,12,32,342)\end{array}$ & $\begin{array}{c}(19,58,125,185,230,272,339) \\
(0,0,0,0,0,0,0)\end{array}$ & 11 & $\begin{array}{l}\text { Gantry } \\
\text { Couch }\end{array}$ & $\begin{array}{c}(9,92,127,163,214,266,317) \\
(64,0,0,0,32,64,0)\end{array}$ & $\begin{array}{c}(8,137,207,229,268,293,353) \\
(0,0,0,0,0,0,0)\end{array}$ \\
\hline 2 & $\begin{array}{l}\text { Gantry } \\
\text { Couch }\end{array}$ & $\begin{array}{l}(7,70,119,151,226,270,351) \\
(332,324,344,290,18,30,64)\end{array}$ & $\begin{array}{c}(75,101,126,161,193,230,356) \\
(0,0,0,0,0,0,0)\end{array}$ & 12 & $\begin{array}{l}\text { Gantry } \\
\text { Couch }\end{array}$ & $\begin{array}{c}(10,91,125,164,200,237,351) \\
(0,0,8,8,32,352,352)\end{array}$ & $\begin{array}{c}(10,92,132,162,205,237,352) \\
(0,0,0,0,0,0,0)\end{array}$ \\
\hline 3 & $\begin{array}{l}\text { Gantry } \\
\text { Couch }\end{array}$ & $\begin{array}{l}(8,49,121,164,256,283,325) \\
(336,334,352,286,348,32,4)\end{array}$ & $\begin{array}{c}(15,86,114,178,235,319,337) \\
(0,0,0,0,0,0,0)\end{array}$ & 13 & $\begin{array}{l}\text { Gantry } \\
\text { Couch }\end{array}$ & $\begin{array}{c}(4,71,123,182,230,285,329) \\
(64,328,8,0,24,8,56)\end{array}$ & $\begin{array}{c}(2,43,65,92,133,301,339) \\
(0,0,0,0,0,0,0)\end{array}$ \\
\hline 4 & $\begin{array}{l}\text { Gantry } \\
\text { Couch }\end{array}$ & $\begin{array}{c}(48,89,139,202,236,293,353) \\
(28,354,2,78,354,32,48) \\
\end{array}$ & $\begin{array}{c}(12,89,127,176,230,277,339) \\
(0,0,0,0,0,0,0) \\
\end{array}$ & 14 & $\begin{array}{l}\text { Gantry } \\
\text { Couch }\end{array}$ & $\begin{array}{c}(7,68,115,131,214,268,347) \\
(354,330,6,0,32,2,0)\end{array}$ & $\begin{array}{c}(35,60,111,129,184,266,347) \\
(0,0,0,0,0,0,0)\end{array}$ \\
\hline 5 & $\begin{array}{l}\text { Gantry } \\
\text { Couch }\end{array}$ & $\begin{array}{c}(50,109,153,190,264,323,359) \\
(0,18,312,64,4,14,344)\end{array}$ & $\begin{array}{c}(25,59,84,113,169,186,325) \\
(0,0,0,0,0,0,0)\end{array}$ & 15 & $\begin{array}{l}\text { Gantry } \\
\text { Couch }\end{array}$ & $\begin{array}{c}(20,71,133,174,224,277,329) \\
(64,300,332,344,0,0,328)\end{array}$ & $\begin{array}{c}(8,91,139,212,232,281,347) \\
(0,0,0,0,0,0,0)\end{array}$ \\
\hline 6 & $\begin{array}{l}\text { Gantry } \\
\text { Couch }\end{array}$ & $\begin{array}{c}(10,79,131,234,236,285,331) \\
(346,2,344,66,0,358,292)\end{array}$ & $\begin{array}{c}(73,115,139,168,195,233,274) \\
(0,0,0,0,0,0,0)\end{array}$ & 16 & $\begin{array}{l}\text { Gantry } \\
\text { Couch }\end{array}$ & $\begin{array}{c}(21,64,121,175,246,278,347) \\
(72,328,8,288,54,34,46)\end{array}$ & $\begin{array}{c}(84,109,165,194,230,319,359) \\
(0,0,0,0,0,0,0)\end{array}$ \\
\hline 7 & $\begin{array}{l}\text { Gantry } \\
\text { Couch }\end{array}$ & $\begin{array}{c}(38,77,131,180,236,271,341) \\
(90,332,0,280,24,8,328)\end{array}$ & $\begin{array}{c}(105,135,200,234,265,335,358) \\
(0,0,0,0,0,0,0)\end{array}$ & 17 & $\begin{array}{l}\text { Gantry } \\
\text { Couch }\end{array}$ & $\begin{array}{l}(24,89,149,188,240,275,301) \\
(358,356,324,358,358,40,16)\end{array}$ & $\begin{array}{c}(24,87,131,188,240,297,351) \\
(0,0,0,0,0,0,0)\end{array}$ \\
\hline 8 & $\begin{array}{l}\text { Gantry } \\
\text { Couch }\end{array}$ & $\begin{array}{c}(6,79,125,196,232,283,345) \\
(28,312,336,76,12,34,2)\end{array}$ & $\begin{array}{c}(18,52,120,178,265,338,351) \\
(0,0,0,0,0,0,0)\end{array}$ & 18 & $\begin{array}{l}\text { Gantry } \\
\text { Couch }\end{array}$ & $\begin{array}{c}(17,62,109,129,216,274,333) \\
(76,2,326,296,2,8,36)\end{array}$ & $\begin{array}{c}(14,73,123,178,228,277,333) \\
(0,0,0,0,0,0,0)\end{array}$ \\
\hline 9 & $\begin{array}{l}\text { Gantry } \\
\text { Couch }\end{array}$ & $\begin{array}{c}(40,91,133,184,224,263,357) \\
(84,326,346,64,24,40,48)\end{array}$ & $\begin{array}{c}(76,102,132,156,200,225,358) \\
(0,0,0,0,0,0,0)\end{array}$ & 19 & $\begin{array}{l}\text { Gantry } \\
\text { Couch }\end{array}$ & $\begin{array}{c}(28,95,173,194,242,299,349) \\
(334,334,328,16,352,34,8)\end{array}$ & $\begin{array}{c}(5,96,132,170,194,226,250) \\
(0,0,0,0,0,0,0)\end{array}$ \\
\hline 10 & $\begin{array}{l}\text { Gantry } \\
\text { Couch }\end{array}$ & $\begin{array}{c}(23,92,125,181,228,264,329) \\
(72,352,8,340,0,58,2)\end{array}$ & $\begin{array}{c}(38,89,139,194,244,297,353) \\
(0,0,0,0,0,0,0)\end{array}$ & 20 & $\begin{array}{l}\text { Gantry } \\
\text { Couch }\end{array}$ & $\begin{array}{c}(22,79,129,158,234,295,357) \\
(64,310,352,312,6,38,88)\end{array}$ & $\begin{array}{c}(16,81,131,164,236,295,355) \\
(0,0,0,0,0,0,0)\end{array}$ \\
\hline
\end{tabular}

an automated way, a single Pareto optimal IMRT plan for a given number of beams ${ }^{34}$. This hierarchical algorithm is organized around two phases. In the first phase, following the priorities of the wish-list, the objectives are optimized without violating the hard constraints. After the optimization of each objective, a new hard constraint embedding the current optimal objective outcome is added to be considered in the optimization of the lower level objectives. This strategy assures that outcomes of higher priority objectives are not jeopardized by the optimization of lower priority objectives. At the end of the first phase, the treatment plan obtained fulfills all hard constraints of the wish-list and simultaneously attain a value for each objective that is equal to its goal or higher if the constraints, including constraints added from higher priority objectives, prevent a better outcome. In the second phase, all objectives, except $L T C P$ objectives, are fully optimized following their wish-list prioritization. For a detailed description of the $2 p \epsilon c$ algorithm see Breedveld et al. ${ }^{34}$.

\section{Computational tests}

The computational tests were performed on a modern 8-core workstation. Erasmus-iCycle, an in-house optimization platform written in MATLAB, developed at Erasmus MC Cancer Institute in Rotterdam ${ }^{5,34,35}$, was used to embed our parallel multistart BAO algorithm. The Erasmus-iCycle multicriteria fluence map optimizer, $2 p \epsilon c$, makes full use of multi-threaded computing ${ }^{34}$ and was used to obtain the optimal value of the FMO problem. The choice of Erasmus-iCycle to embed our BAO optimization is justified by the reliability of its multicriteria fluence map optimizer to achieve good treatment plans for complex cases as nasopharyngeal tumors. Nevertheless, the FMO is treated as a black-box. Thus, other FMO algorithms can be easily coupled with this BAO strategy. It should be highlighted that, unlike our previous $\mathrm{BAO}$ studies that only considered a limited number of structures, all structures of the complex cases tested were included in this study.

A set of twenty nasopharyngeal tumors treated at IPOC was used retrospectively to test the parallel multistart noncoplanar BAO framework. The mean (minimum-maximum) volume of the PTV-T is $59.4(20.6-106.5) \mathrm{cm}^{3}$ and the mean (minimum-maximum) volume of the PTV-N is $525.9(303.8-705.4) \mathrm{cm}^{3}$. Treatment plans with seven noncoplanar beam orientations, obtained using the parallel multistart framework and denoted $B A O_{n c}$, were compared against treatment plans with seven coplanar beam orientations, obtained using the parallel multistart framework and denoted $B A O_{c}$. Gantry and couch angles obtained for $B A O_{n c}$ and $B A O_{c}$ treatment plans are depicted in Table II. These treatment plans were compared against treatment plans with seven equispaced coplanar beam ensembles, denoted Equi, commonly used at IPOC and in clinical practice to treat nasopharyngeal cases ${ }^{17}$ and used here as benchmark. All treatment plans were generated and compared using Erasmus-iCycle.

In our noncoplanar approach, for computational time efficiency, only the most promising regions have active local search by choosing a threshold, $p=0.1$, related to the best solution found so far. This threshold was determined in

preliminary tests and corresponds to the smaller value of $p>0$ that leads to similar results obtained by considering all regions with active local search. The PSM algorithm implemented considered the directions of the maximal positive basis $([I-I])$, an initial step-size of $\sigma^{1}=2^{5}=32$ and a minimal step-size value of one which defined 
the stopping criteria. With this choice of initial step-size, since step-size is halved at unsuccessful iterations, all beam directions considered are integer until the termination criteria when the step-size becomes inferior to one. For a matter of computational time efficiency, no trial points were computed in the search step. For the coplanar $\mathrm{BAO}, B A O_{c}$, the mean number of FMO evaluations was 2019 corresponding to a mean computational time of 12.4 h. For the noncoplanar BAO, $B A O_{n c}$, the mean number of FMO evaluations was 2840 corresponding to a mean computational time of $18.3 \mathrm{~h}$. Most of the computational time is spent on the computation of the expensive (in terms of time) objective function value (FMO). For each beam ensemble, the FMO performed by the Erasmus-iCycle FMO in dosimetric computation.

\section{RESULTS}

Target metrics computed for the different treatment plans included the tumor coverage, conformity, and homogeneity, corresponding to output values of Erasmus-iCycle FMO optimizer. Tumor coverage is computed as the ratio between the volume of PTV receiving at least $95 \%$ of the prescribed dose and the volume of PTV. The ratio between the volume of PTV receiving at least $95 \%$ of the prescribed dose and the volume outside PTV receiving at least $95 \%$ of the prescribed dose corresponds to conformity. Homogeneity is calculated as the ratio between the dose received by $95 \%$ of the volume and the dose received by $5 \%$ of the volume. The mean values of PTV-T tumor coverage, conformity and homogeneity for $B A O_{n c}$ treatment plans was $0.98,0.67$, and 0.90 , respectively. The mean values of PTV-N tumor coverage, conformity and homogeneity for $B A O_{n c}$ treatment plans was $0.99,0.61$, and 0.82 , respectively. These metrics presented very small differences for $B A O_{n c}, B A O_{c}$, and Equi treatment plans.

Organ sparing results, i.e. mean and/or maximum doses, depending if the organ has a parallel or serial architecture, are displayed for the twenty cases in Fig. 3. For spinal cord and brainstem, serial organs, the maximum dose is displayed. For these structures, it can be verified that $B A O_{n c}$ clearly obtained the best sparing results. Compared to Equi treatment plans, $B A O_{n c}$ obtained an improved average sparing for spinal cord and brainstem of $1.8 \mathrm{~Gy}$ and 7.3 Gy, respectively. These results are particularly impressive for brainstem, and may have added importance, e.g. for re-irradiation cases, since maximum doses are many times close to the tolerance dose. $B A O_{c}$ manage to obtain an improved average sparing for spinal cord and brainstem of $0.3 \mathrm{~Gy}$ and $2.5 \mathrm{~Gy}$, respectively, compared to Equi treatment plans. For parotids, oral cavity and thyroid, parallel organs, the mean dose is displayed. Again, $B A O_{n c}$ sparing for left parotid, right parotid, oral cavity, and thyroid of 3.9 Gy, 3.0 Gy, 6.1 Gy, and 2.1 Gy, respectively Sparing of salivary glands may prevent xerostomia, a common complication of radiation therapy for head-and-neck cancer cases. While parotids are the largest of the three salivary glands, submandibular glands, the second largest salivary glands, and the sublingual glands are very important in saliva production. At IPOC, both the submandibular

for both parotids and oral cavity is of the utmost interest. The sparing results of thyroid are the least impressive at first sight. However, looking at thyroid priority on the wish-list displayed in Table I, this result highlights the capacity of sparing low level priority structures as well. $B A O_{c}$ treatment plans achieve more modest improvements when compared to Equi treatment plans. In average, $B A O_{c}$ treatment plans achieve a mean dose irradiation reduction on the left parotid, right parotid, oral cavity, and thyroid of 1.4 Gy, 1.2 Gy, 3.9 Gy, and 1.3 Gy, respectively. For space reasons, other lower level wish-list structures results are not displayed. Nevertheless, $B A O_{n c}$ treatment plans outperform the Equi treatment plans for other structures. For instance, for esophagus and larynx, $B A O_{n c}$ treatment plans obtained, in average, a mean dose irradiation reduction of 1.7 Gy and 3.1 Gy, respectively.

In clinical practice, plans' quality is also assessed by cumulative dose-volume histograms (DVHs). For illustration, DVH results for a typical patient (ninth) are displayed in Fig. 4. For clarity, tumor volumes, spinal cord, brainstem, parotids, oral cavity, and thyroid are displayed in two separated figures. The DVH curves show that for a similar tumor coverage, a better organ sparing is obtained by the treatment plan using the optimized noncoplanar beam directions.

It is important to remark that clinical decision is made case by case. Thus, as important as average results, it is lead to clearly better treatment plans for some cases at free cost, i.e. in an automated way, then BAO purpose is fully attained.

Iterative BAO, considering noncoplanar beam orientations for a $5^{\circ}$ angular spacing, has been used to generate an ideal $4 \pi$ plan that would correspond to a theoretical upper limit of the treatment plan quality ${ }^{12}$. Two treatment

plans with seven coplanar and with seven noncoplanar beam orientations were obtained using iterative BAO and considering beam orientations for a $5^{\circ}$ angular spacing. These plans were compared with $B A O_{n c}$ and $B A O_{c}$. Since noncoplanar iterative BAO requires a huge number of FMO calculations, this comparison was only performed for the 


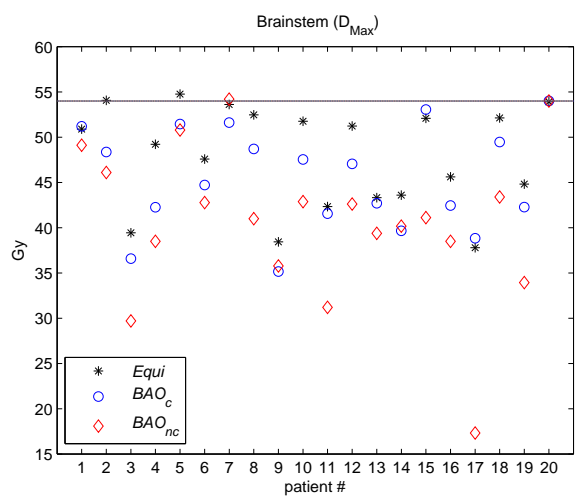

(a)

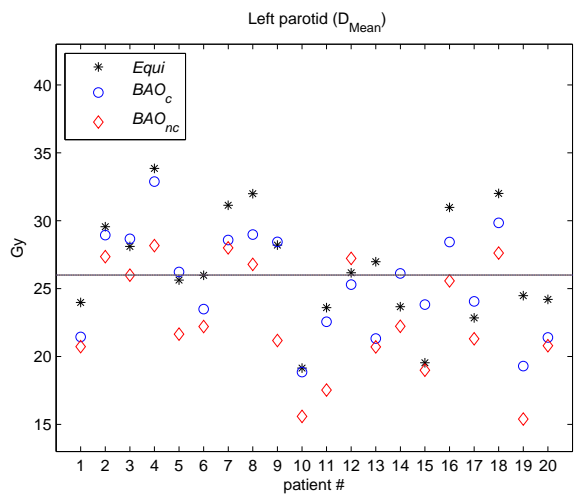

(c)

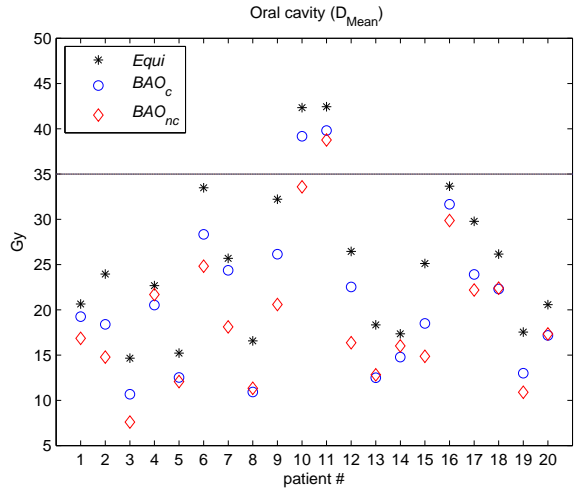

(e)

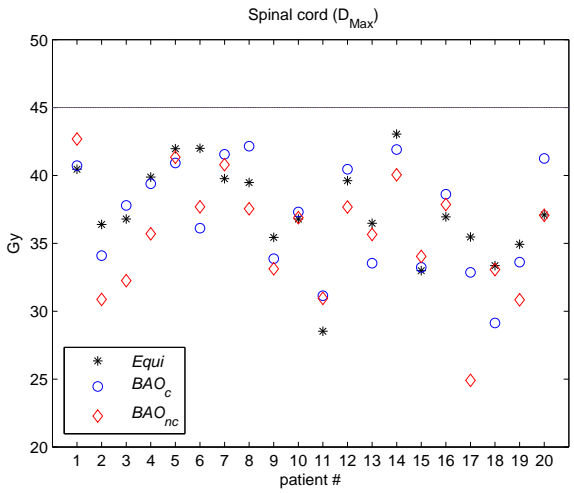

(b)

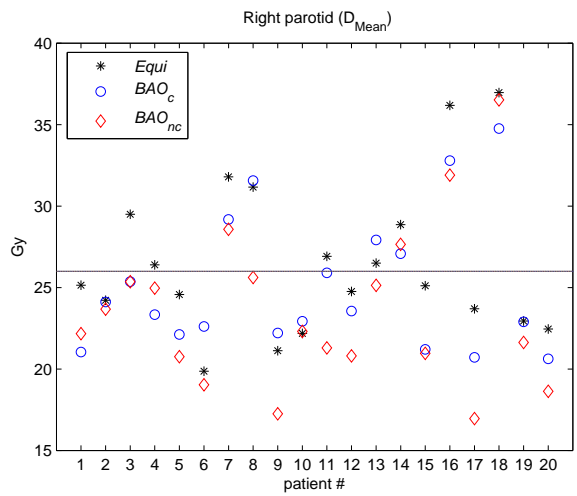

(d)

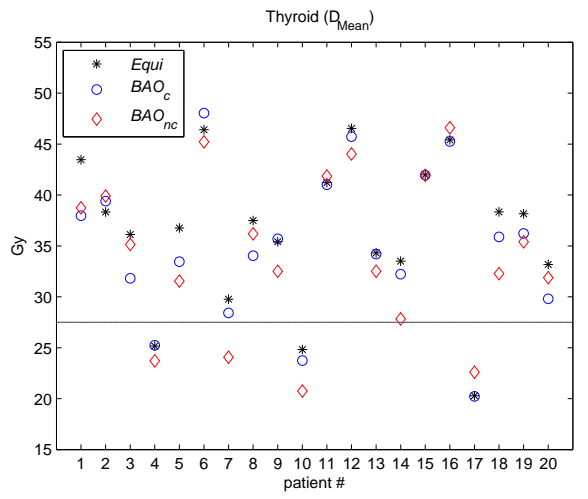

(f)

FIG. 3. Comparison of organ sparing metrics obtained by Equi, $B A O_{c}$, and $B A O_{n c}$ treatment plans. The horizontal lines displayed represent the tolerance (mean or maximum) dose for each structure.

ninth patient, the one corresponding to Fig. 4. Comparison of the two strategies should consider two criteria. While the main goal is to obtain the best objective function value possible (quality), another important goal is to obtain a good solution as fast as possible (time). Fig. 5 display the solutions obtained by the different approaches in terms of quality and time. It can be seen that the results in terms of objective function value clearly favor the multistart approach and the use of noncoplanar directions. In terms of FMO evaluations, a small increase is verified for the multistart strategy when adding noncoplanar directions while a drastic increase is verified for the iterative strategy when adding noncoplanar beams. 

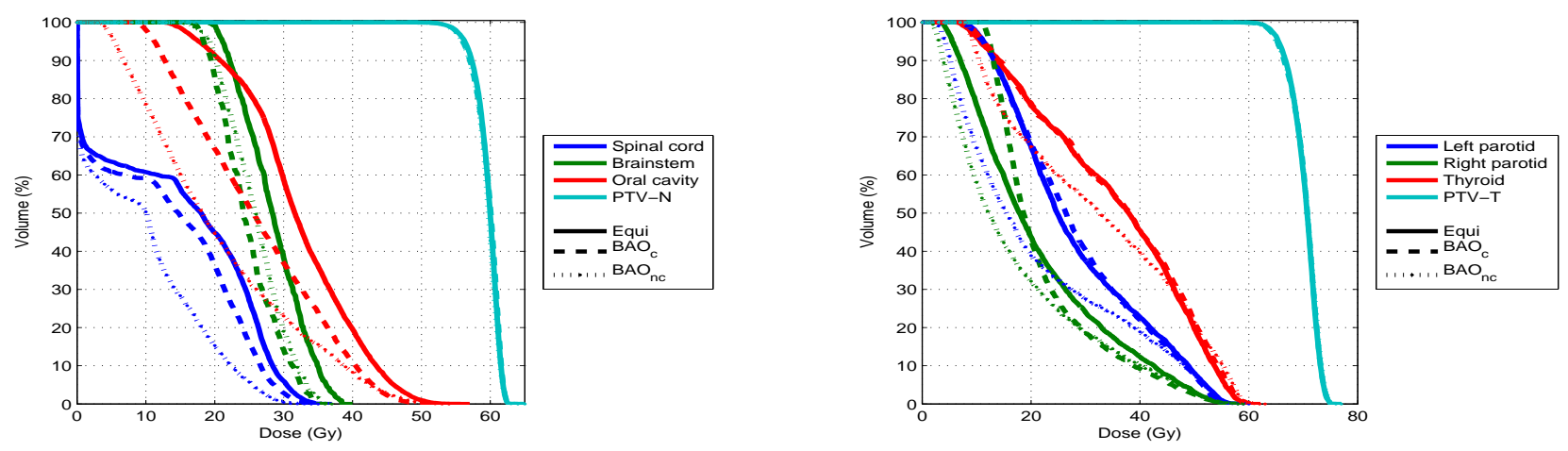

FIG. 4. Cumulative dose volume histogram comparing the results obtained by BAO and Equi for one patient.

\section{DISCUSSION}

In this study we propose a novel approach for the resolution of the noncoplanar BAO problem using a different methodological approach. The proposed approach presents an alternative to other known approaches for IMRT treatment planning. A multistart derivative-free framework was sketched and tested using a set of twenty clinical nasopharyngeal tumor cases. Multistart approaches are suitable for the highly non-convex BAO problem since they combine a global strategy for sampling the search space with a local strategy for improving the sampled solutions. The global strategy designed provide a non-random strategy for distributing the initial beam ensembles on the BAO continuous reduced search space while the local strategy using PSM proved, in previous works, to be successful in improving locally beam angle ensembles, requiring few function evaluations and avoiding local entrapment.

For the twenty nasopharyngeal clinical cases tested, comparisons between the different treatment plans clearly favor plans obtained by the multistart derivative-free approach with noncoplanar beam directions. The considerable plan quality improvements relatively to the benchmark plan, using seven coplanar equispaced directions, were obtained considering a large number of structures and using a reliable IMRT platform, Erasmus-iCycle, capable of obtaining good treatment plans for complex cases as nasopharyngeal tumors. For similar target coverage, plans using optimized beam directions improved high level wish-list structures, in particular brainstem, but also lower level wish-list structures including salivary glands. Considering different wish-lists priorities may lead to different levels of sparing for the different structures. That tunning process should be made for each institution for the different tumor sites. Furthermore, since FMO is treated as a black-box, other FMO algorithms can be easily coupled with this parallel multistart BAO framework.

For one nasopharyngeal clinical case, the multistart derivative-free approach with noncoplanar beam directions was also compared with iterative BAO considering noncoplanar beam orientations for a $5^{\circ}$ angular spacing. This latter approach, $4 \pi$, has been used as theoretical upper limit of the treatment plan quality ${ }^{12}$. The results for the nasopharyngeal clinical case tested imply that $4 \pi$ is not a theoretical upper limit of the treatment plan quality and highlight that the frontier of the best expectations can be overcome. This might indicate that BAO potential is yet far from being fully explored both in terms of quality and time.

In this multistart noncoplanar approach several strategies were embedded to reduce, as much as possible, the number of function evaluations leading to a good compromise between the number of function evaluations and the quality of the solutions. The strategies drafted included the non-random strategy sketched to take advantage of a reduced search space, the use of a generalization of regions of attraction of a local minimum and the limitation of local searches to the most promising regions. Other approaches to accelerate BAO have been made by reducing the arithmetic load of $\mathrm{FMO}^{5}$ or by an early termination of $\mathrm{FMO}^{36}$. These strategies accelerate the computation of a beam's ensemble quality measure rather than the BAO process itself and can thus be promptly incorporated in any strategy that uses the optimal FMO problem value to drive the BAO procedure. Finally, the computational burden of integrated beam orientation and fluence optimization is manageable using the current generation of calculation platforms, which will only become faster. 


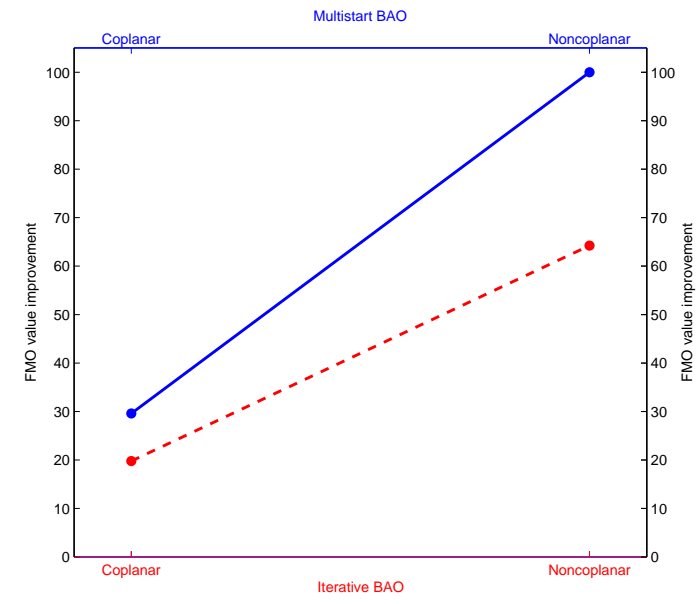

(a)

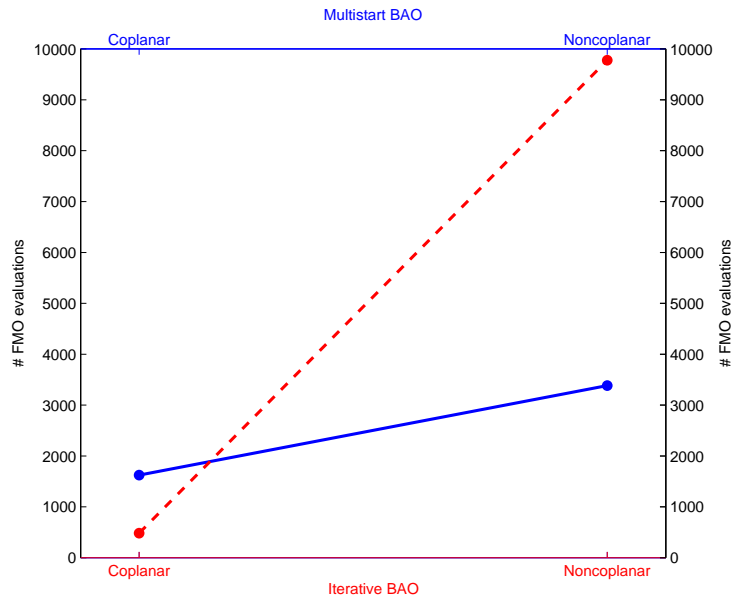

(b)

FIG. 5. Relative FMO improvement comparing the benchmark beam ensemble, Equi, (0\% improvement) and the best treatment plan, $B A O_{n c}$, (100\% improvement) - 5(a) and corresponding number of FMO evaluations required to obtain the corresponding solutions $-5(\mathrm{~b})$.

\section{ACKNOWLEDGMENTS}

This work has been supported by the Fundação para a Ciência e a Tecnologia (FCT) under project grant UID/MULTI/00308/2013. We would like to show gratitude to Ben Heijmen and Sebastiaan Breedveld for giving permission and helping us to install Erasmus-iCycle.

The authors have no relevant conflicts of interest to disclose.

[1] Bangert, M., Ziegenhein, P., Oelfke, U.: Comparison of beam angle selection strategies for intracranial imrt. Med. Phys. 40, 011716 (2013).

[2] Bangert, M., Oelfke, U.: Spherical cluster analysis for beam angle optimization in intensity-modulated radiation therapy treatment planning. Phys. Med. Biol. 55, 6023-37 (2010).

[3] Rossi, L., Breedveld, S., Aluwini, S., Heijmen, B.: Noncoplanar Beam Angle Class Solutions to Replace Time-Consuming Patient-Specific Beam Angle Optimization in Robotic Prostate Stereotactic Body Radiation Therapy Int. J. Radiat. Oncol., Biol., Phys. 92, 762-70 (2015).

[4] Sharfo, A-W., Voet, P., Breedveld, S., Mens, J-W., Hoogeman, M., Heijmen, B.: Comparison of VMAT and IMRT strategies for cervical cancer patients using automated planning. Radiother. Oncol. 114, 395-401 (2015).

[5] Breedveld, S., Storchi, P.R.M., Voet, P.W.J.,Heijmen, B.J.M.: iCycle: Integrated, multicriterial beam angle, and profile optimization for generation of coplanar and noncoplanar IMRT plans. Med. Phys. 39, 951-63 (2012).

[6] Rossi, L., Breedveld, S., Heijmen, B.J.M., Voet, P.W.J., Lanconelli, N.,Aluwini, S.: On the beam direction search space in computerized non-coplanar beam angle optimization for IMRT prostate SBRT. Phys. Med. Biol. 57, 5441 (2012).

[7] Voet, P.W.J., Breedveld, S., Dirkx,M.L.P., Levendag, P.C. ,Heijmen, B.J.M.: Integrated multicriterial optimization of beam angles and intensity profiles for coplanar and noncoplanar head and neck IMRT and implications for VMAT. Med. Phys. 39, 4858-65 (2012).

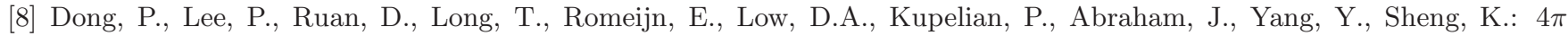
noncoplanar stereotactic body radiation therapy for centrally located or larger lung tumors. Int. J. Radiat. Oncol., Biol., Phys. 86, 407-13 (2013).

[9] Llacer, J., Li, S., Agazaryan, N., Promberger, C., Solberg, T.D.: Noncoplanar automatic beam orientation selection in cranial IMRT: a practical methodology. Phys. Med. Biol. 54, 1337-68 (2009).

[10] Mišić, V.V., Aleman, D.M., Sharpe, M.B.: Neighborhood search approaches to non-coplanar beam orientation optimization for total marrow irradiation using IMRT. Eur. J. Oper. Res. 205, 522-7 (2010).

[11] Papp, D., Bortfeld, T., Unkelbach, J.: A modular approach to intensity-modulated arc therapy optimization with noncoplanar trajectories. Phys. Med. Biol. 60, 5179-98 (2015).

[12] Wild, E., Bangert, M., Nill, S., Oelfke, U.: Noncoplanar VMAT for nasopharyngeal tumors: Plan quality versus treatment 
time. Med. Phys. 42, 2157-68 (2015).

[13] Sheng, K., Shepard, D.M., Orton, C.G.: Noncoplanar beams improve dosimetry quality for extracranial intensity modulated radiotherapy and should be used more extensively. Med. Phys. 42, 531-3 (2015).

[14] Woods, K., Nguyen, D., Tran, A., Yu, V.Y., Cao, M., Niu, T., Lee, P., Sheng, K.: Viability of Non-Coplanar VMAT for Liver SBRT as Compared to Coplanar VMAT and Beam Orientation Optimized $4 \pi$ IMRT. Advances in Radiation Oncology (2016).

[15] Craft, D.: Local beam angle optimization with linear programming and gradient search. Phys. Med. Biol. 52, 127-35 (2007).

[16] Pugachev, A., Xing, L.: Pseudo beam's-eye-view as applied to beam orientation selection in intensity-modulated radiation therapy. Int. J. Radiat. Oncol. Biol. Phys. 51, 1361-70 (2001)

[17] Aleman, D.M., Kumar, A., Ahuja, R.K., Romeijn, H.E., Dempsey, J.F.: Neighborhood search approaches to beam orientation optimization in intensity modulated radiation therapy treatment planning. J. Global Optim. 42, 587-607 (2008)

[18] Lim, G.J., Cao, W.: A two-phase method for selecting IMRT treatment beam angles: Branch-and-Prune and local neighborhood search. Eur. J. Oper. Res. 217, 609-18 (2012)

[19] Bertsimas, D., Cacchiani, V., Craft, D., Nohadani, O.: A hybrid approach to beam angle optimization in intensitymodulated radiation therapy. Comput. Oper. Res. 40, 2187-97 (2013)

[20] Dias, J., Rocha, H., Ferreira, B.C., Lopes, M.C.: A genetic algorithm with neural network fitness function evaluation for IMRT beam angle optimization. Cent. Eur. J. Oper. Res. 22, 431-55 (2014).

[21] Dias, J., Rocha, H., Ferreira, B.C., Lopes, M.C.: Simulated annealing applied to IMRT beam angle optimization: A computational study. Physica Medica 31, 747-56 (2015).

[22] Bangert, M., Ziegenhein, P., Oelfke, U.: Characterizing the combinatorial beam angle selection problem. Phys. Med. Biol. 57, 6707-23 (2012).

[23] Martí, R., Resende, M.G.C., Ribeiro, C.C.: multistart methods for combinatorial optimization. Eur. J. Oper. Res. 226 $1-8(2013)$.

[24] Rocha, H., Dias. J., Ferreira, B.C., Lopes, M.C.: Selection of intensity modulated radiation therapy treatment beam directions using radial basis functions within a pattern search methods framework. J. Glob. Optim. 57 1065-89 (2013).

[25] Rocha, H., Dias. J., Ferreira, B.C., Lopes, M.C.: Beam angle optimization for intensity-modulated radiation therapy using a guided pattern search method. Phys. Med. Biol. 58 2939-53 (2013).

[26] Rocha, H., Dias. J., Ferreira, B.C., Lopes, M.C.: Pattern search methods framework for beam angle optimization in radiotherapy design. Appl. Math. Comput. 219 10853-65 (2013).

[27] Alberto, P., Nogueira, F., Rocha, H., Vicente, L.N.: Pattern search methods for user-provided points: Application to molecular geometry problems. SIAM J. Optim. 14, 1216-36 (2004).

[28] Davis, C.: Theory of positive linear dependence. Am. J. Math. 76, 733-46 (1954).

[29] Torczon, V.: On the convergence of pattern search algorithms. SIAM J. Optim. 7, 1-25 (1997).

[30] Voglis, C., Lagaris, I.: Towards "ideal multistart". A stochastic approach for locating the minima of a continuous function inside a bounded domain. App. Math. Comput. 213 1404-1415 (2009).

[31] Rinnooy Kan, A., Timmer, G.: Stochastic global optimization problems Part II: Multi level methods. Mathematical Programming, 39 57-78 (1987).

[32] Craft, D., Halabi, T., Shih, H., Bortfeld, T.: Approximating convex Pareto surfaces in multiobjective radiotherapy planning. Med. Phys. 33, 3399-3407 (2006)

[33] Monz, M., Kufer, K.H., Bortfeld, T.R., Thieke, C.: Pareto navigation Algorithmic foundation of interactive multi-criteria IMRT planning. Phys. Med. Biol. 53, 985-998 (2008)

[34] Breedveld, S., Storchi, P., Keijzer, M., Heemink, A.W., Heijmen, B.: A novel approach to multi-criteria inverse planning for IMRT. Phys. Med. Biol. 52, 6339-6353 (2007)

[35] Breedveld, S., Storchi, P., Heijmen, B.: The equivalence of multicriteria methods for radiotherapy plan optimization. Phys. Med. Biol. 54, 7199-7209 (2009)

[36] Bangert, M., Unkelbach, J.: Accelerated iterative beam angle selection in IMRT. Med. Phys. 43, 1073-1082 (2016)

[37] See supplementary material at [URL will be inserted by AIP] for more detailed dosimetry results and DVHs. 